\title{
Neoextractivismo. Debates y conflictos en los países con gobiernos progresistas en Suramérica'
}

Recibido: 18/10/2016

Aprobado: 21/11/2016

Edgardo Lander

Universidad Central de Venezuela

<elandel@yahoo.com >

\section{RESUMEN}

La especialización en la producción de materias primas, lejos de permitir una acumulación que garantice la inversión en alternativas al extractivismo, tiende a bloquear la posibilidad de otras actividades generando procesos de desindustrialización en el continente, y el deterioro de la soberanía alimentaria. Con esto no se están construyendo alternativas al capitalismo, ni siquiera poniéndole obstáculos, sino alimentando su insaciable maquinaria depredadora.

Palabras Clave: Neoextractivismo, conflictos, gobiernos progresistas

\section{Neoextractivism. Debates and conflicts in countries with progressive governments in South America}

\begin{abstract}
The specialization in the production of raw materials, far from allowing an accumulation that guarantees the investment in alternatives to the extractivism, tends to block the possibility of other activities generating processes of deindustrialization in the continent, and the deterioration of the food sovereignty. With this no alternatives to capitalism are being built, not even putting obstacles, but feeding their insatiable predatory machinery
\end{abstract}

KEYWORDS: Neo-extractivism, conflicts, progressive governments, South America

1 Una versión inicial de este texto fue presentada en el "Coloquio Especial sobre Ecología Política. Extractivismo y modelos de desarrollo en América Latina y el Caribe”, en el contexto de la VII Conferencia Latinoamericana y Caribeña de Ciencias Sociales del Consejo Latinoamericano de Ciencias Sociales (CLACSO) celebrada en Medellín en noviembre, 2015. 


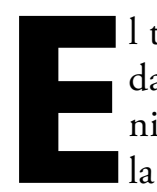

1 tema del extractivismo ha generado profundas divisiones en el seno de la izquierda, organizaciones y movimientos populares durante la última década. Esto es expresión, entre otras cosas, de visiones diferentes de la sociedad a la cual se aspira, concepciones encontradas en torno al carácter de la actual crisis civilizatoria, evaluaciones no compartidas sobre lo que fue el socialismo del siglo XX y, en consecuencia, de prioridades diferentes que unos y otros sectores le otorgan a las principales dimensiones o ejes del cambio requerido en estas sociedades.

Es posible caracterizar las partes de esas confrontaciones muy esquemáticamente en los siguientes términos. Por un lado, quienes - por lo menos para la primera etapa de los procesos de cambio- le otorgan prioridad en las agendas de transformación al antiimperialismo, al rechazo a las políticas económicas del neoliberalismo, a la recuperación del Estado, a la soberanía nacional, la superación a corto plazo de la pobreza/desigualdad y al crecimiento económico. Tienden en general a otorgarle menor prioridad o tener una visión poco problematizadora en torno a asuntos como el patriarcado, la interculturalidad, las autonomías territoriales o las implicaciones ambientales y estratégicas de un modelo productivo centrado en el extractivismo.

Por el otro, una variedad de perspectivas que, sin negar la importancia de todo lo anterior, afirman la necesidad de confrontar igualmente, y en forma simultánea, el racismo, el patriarcado, la colonialidad y el antropocentrismo característicos del capitalismo. Asumen que si no se detienen a muy corto plazo las dinámicas de destrucción de la vida en el planeta y de la amplia gama de culturas que caracterizan a la humanidad, ninguna transformación en una dirección democrática emancipadora será posible. Desde estas perspectivas se asume que los instrumentos teóricos y las políticas que en el siglo pasado parecían suficientes para enfrentar al capitalismo, centrados en las relaciones de clase, las formas de propiedad, y el papel rector del Estado, ya no son suficientes para el mundo en que vivimos. Hoy tenemos que confrontarnos no solo a las formas de dominación económica y política del capitalismo, sino igualmente a la geo-cultura de la modernidad. Se trata de retos propiamente civilizatorios y por ello, mucho más exigentes: un sistema global patriarcal que está aceleradamente destruyendo tanto las condiciones materiales que hacen posible la vida en el planeta, como la extraordinaria diversidad de las memorias y culturas de los pueblos que lo habitan. Se tienen, en consecuencia, miradas radicalmente críticas en torno a la posibilidad de que la acentuación del modelo extractivista pueda considerarse como una vía para superar el capitalismo.

En estas oposiciones hay igualmente diferencias profundas en torno a los agentes de los procesos de transformación. En el primer bloque se le asigna una clara prioridad al Estado como agente rector de los procesos de cambio, mientras para el otro bloque las transformaciones requeridas pasan prioritariamente por movimientos y organizaciones sociales, pueblos y comunidades, por las múltiples expresiones del tejido de la sociedad.

Estas divergencias en las interpretaciones de los procesos políticos de estos años atraviesan igualmente a la academia latinoamericana.

Los gobiernos "progresistas" y sus defensores argumentan que es (o era) necesario aprovechar el contexto de la elevada demanda y precio de los commodities para acumular los recursos requeridos con el fin de realizar las inversiones sociales, productivas y de infraestructura que permitiesen, en una fase posterior, superar el extractivismo. Ello pasaría, necesariamente, por un mayor control estatal sobre la explotación de las materias primas, ya sea mediante nacionalizaciones o mediante mayores cargas impositivas, para lograr una mayor participación en la renta que antes había tenido a las corporaciones transnacionales como principales beneficiarias.

En el contexto internacional favorable a los exportadores de commodities de la última década, las políticas de ampliación de las actividades extractivas y de mayor participación del Estado en los ingresos provenientes de éstas, hicieron posibles importantes logros que estos gobiernos pueden reivindicar. Se dio, durante varios años, un crecimiento económico sostenido. Después de un largo período de déficits, entre los años 2002 y el 2007, América Latina en su conjunto tuvo saldos de cuenta corriente favorables. Entre el año 2003 y el año 2012 la deuda externa total como porcentaje del producto se redujo a menos de la mitad. La inversión extranjera directa creció aceleradamente. ${ }^{1}$ En términos geopolíticos, estas condiciones económicas favorables permitieron mayores niveles de autonomía. Con una mayor diversificación geográfica del comercio exterior

1 CEPAL. Anuario Estadístico de América Latina y el Caribe 2013, Santiago de Chile, 2013. 
y el acceso a otras fuentes de crédito, se redujo la elevada dependencia que antes se tenía con relación a los Estados Unidos y la Unión Europea. Las balanzas de pagos positivas permitieron pagar deudas externas y librarse de la tutela de las instituciones Bretton Woods. Se acumularon reservas internacionales. Después de la derrota del ALCA se dieron pasos hacia nuevos mecanismos de integración regional como el ALBA, UNASUR y CELAC. En lo fundamental, el continente dejó de ser el patio trasero de los Estados Unidos.

El sostenido incremento de los ingresos fiscales permitió inversiones masivas en programas sociales como las misiones en Venezuela y el programa Bolsa Familia en Brasil, que contribuyeron a sacar a millones de personas de la pobreza. En todos estos países mejoró el acceso de los sectores populares a los servicios educativos, de salud y a la seguridad social, e incluso se dio una cierta reducción de la desigualdad medida en términos de ingreso.

En consecuencia, estos gobiernos contaron con importantes grados de legitimidad y lograron estabilidad política después de años caracterizados por protestas populares, golpes de Estado y gobiernos que no lograban concluir sus períodos constitucionales. Consiguieron -en todos los casos- sucesivas victorias electorales. En Venezuela el gobierno ganó en cada una de las tres elecciones presidenciales que se realizaron desde que Hugo Chávez accedió a la presidencia en el año 1999. El Partido de los Trabajadores en Brasil ganó cuatro elecciones presidenciales sucesivas. El Frente para la Victoria en Argentina y el Frente Amplio de Uruguay ganaron tres elecciones presidenciales seguidas. Evo Morales fue reelecto presidente de Bolivia con 63\% de los votos en octubre del 2014. De acuerdo a las encuestas, Rafael Correa cerró ese ańo con niveles de popularidad personal de entre $70 \mathrm{y}$ $80 \%$ en Ecuador.

Sobre la base de todo esto podría afirmarse que esta apuesta por el crecimiento basado en un modelo productivo extractivo y de reprimarización de las economías ha sido extraordinariamente exitosa.

Sin embargo, como se señaló antes, existen otras miradas, otros criterios, otros horizontes normativos, otras concepciones en torno a las sociedades a las cuales se aspira para evaluar estas experiencias. Si incorporamos estas otras perspectivas llegamos a balances bastante más complejos, mucho más preñados de tensiones y contradicciones.

\section{¿Transformaciones civilizatorias o crecimiento económico capitalista?}

Son muchas las razones por las cuales se generaron expectativas de que América del Sur era la región del planeta en la cual sería posible articular las luchas contra el neoliberalismo y por la superación del capitalismo, con pasos en la dirección de transiciones hacia alternativas civilizatorias al modelo depredador monocultural de crecimiento sin fin característico de la modernidad. En las ampliamente extendidas luchas populares en todo el continente en contra del neoliberalismo y contra lo que llegó a ser su proyecto más perverso, el ALCA, ocuparon un lugar destacado los pueblos indígenas, campesinos y afrodescendientes. La defensa de los territorios, la lucha contra los monocultivos, los transgénicos y la mega minería ocuparon lugares centrales en las agendas de los movimientos. Las nociones del sumak qamaña, suma kawsay de los pueblos indígenas andinos y amazónicos fueron incorporadas a la gramática política de estas luchas. La victoria electoral de diferentes candidatos de izquierda o progresistas fueron posibles gracias a los procesos de acumulación de fuerza transformadora de estas luchas.

Sin embargo, con estos gobiernos se ha profundizado el desarrollismo extractivista y ha crecido el peso del sector primario exportador. Con ello se ha acelerado el suministro de los insumos requeridos para alimentar la lógica depredadora global, y a consolidar al orden capitalista contra el cual se luchaba. Un nuevo consenso continental, lo que Maristella Svampa ha denominado el consenso de los commodities ${ }^{2}$ pasó a ser compartido por todos los gobiernos suramericanos, independientemente de su signo político.

En los debates globales sobre las amenazas representadas por el cambio climático hay un creciente acuerdo en el sentido de que es que indispensable dejar por lo menos $80 \%$ de las reservas probadas de hidrocarburos sin explotar, como condición para reducir a $50 \%$ la probabilidad de que la temperatura media del planeta supere en más de 2 grados centígrados la temperatura existente al comienzo de la revolución industrial, y con ello se pase el umbral de las condiciones que hacen posible la vida humana tal como la conocemos en el planeta Tierra. ¿Cómo es posible que, en conocimiento de esto, el gobierno venezolano, auto-proclamado revolucionario y

2 Maristella Svampa, 'Consenso de los commodities' y lenguajes de valoración en América Latina, Nueva Sociedad, n 244, Buenos Aires, marzo-abril de 2013. 
anticapitalista contemple duplicar la producción petrolera de los 3 millones actuales a 6 millones para el año 2019? ¿Qué puede justificar que el gobierno boliviano abra masivamente áreas protegidas, parques nacionales y territorios de los pueblos indígenas a la explotación de hidrocarburos? ¿Tiene sentido que el gobierno ecuatoriano, después de la experiencia de la masiva devastación socio-ambiental producida por Chevron en la Amazonía ecuatoriana, no solo deje a un lado la propuesta de dejar sin explotar el petróleo del Yasuní, sino que continúe expandiendo la frontera petrolera hacia otros territorios igualmente vulnerables?

Es esta la paradoja, o contradicción, más profunda presente en los países con gobiernos progresistas de Suramérica, especialmente en los casos de Bolivia y Ecuador. Precisamente en el momento en que, por primera vez en la historia de este continente, se han reconocido constitucionalmente los derechos de los pueblos indígenas, llegándose en estos dos países a definir sus Estados como plurinacionales, y se reconocen jurídicamente los derechos de la naturaleza, se está acelerando la expansión de la lógica depredadora-extractivista de desposesión, ocupando/devastando aun aquellos territorios que en los últimos cinco siglos habían permanecido relativamente al margen de la expansión del capital. En estos territorios, en estas nuevas fronteras del capital global, aguas y suelos están siendo contaminados, bosques destruidos, la biodiversidad reducida, poblaciones desplazadas. Los cultivos para el autoconsumo y los mercados locales están siendo sustituidos por monocultivos transgénicos, como es el caso de la soya, ${ }^{3}$ amenazando la soberanía alimentaria. A pesar de taxativos mandatos constitucionales, estos gobiernos no pueden reconocer el derecho de los pueblos indígenas y afrodescendientes a sus territorios tradicionales, ya que son precisamente dichos territorios los que, de acuerdo a sus opciones desarrollistas, tienen que ser sometidos a estas lógicas extractivas. Se acude por el contrario a la criminalización de las resistencias. Este dilema fue reconocido explícitamente en la Declaración de Guayaquil de los presidentes del ALBA en junio del 2013.

... rechazamos la posición extremista de determinados grupos que, bajo la consigna del anti-extractivismo, se oponen sistemáticamente a la explotación de nuestros recursos naturales, exigiendo que esto se

3 Juan Luis Berterretche: «El enclave sojero de Sudamérica» en Millones contra Monsanto, 15/10/2013, <http://millonescontramonsanto.org/ el-enclave-sojero-de-sudamerica/>. pueda hacer solamente sobre la base del consentimiento previo de las personas y comunidades que viven cerca de esa fuente de riqueza. En la práctica, esto supondría la imposibilidad de aprovechar esta alternativa y, en última instancia, comprometería los éxitos alcanzados en materia social y económica. ${ }^{4}$

Frente a las exigencias de prácticas democráticas y el respecto a los derechos de los pueblos indígenas garantizados en las constituciones, estos presidentes claramente optan por lo que denominan "el derecho y la necesidad que tienen nuestros países de aprovechar, de manera responsable y sustentable, sus recursos naturales no renovables", esto es, por el extractivismo.

De esta manera, la principal riqueza históricocultural con la cual contamos en el continente para imaginar y construir alternativas civilizatorias a la hegemonía del capital — precisamente el mundo indígena-campesino- está siendo socavada bajo las banderas del progresismo y del socialismo del siglo XXI. Sería extraordinariamente dramático que dentro de unos pocos ańos se llegara a constatar que las experiencias de los gobiernos progresistas lejos de acercarnos al post-capitalismo, nos han alejado de dicho horizonte.

En términos del impacto devastador de estas actividades sobre los pueblos indígenas, afrodescendientes y campesinos, es indiferente que las corporaciones participantes sean nacionales o extranjeras, occidentales $\mathrm{u}$ orientales, públicas o privadas, o que el discurso que busque legitimarlas sea de mercado o de revolución.

El extractivismo no es, como lo ha argumentado Álvaro García Linera, vicepresidente de Bolivia, una "forma técnica" de producción compatible con cualquier modelo de sociedad. ${ }^{5}$ Es por el contrario, en su mega escala actual, expresión de la profundización de un patrón civilizatorio antropocéntrico, patriarcal y colonial de destrucción de la vida. No es solo un régimen de producción, sino un tipo de sociedad. Además de producir mercancías, el modelo productivo extractivista contribuye a la formación de los agentes sociales involucrados en ese proceso, ${ }^{6}$ genera subjetividades y

4 Declaración del ALBA desde el Pacifico. XII Cumbre de Jefes de Estado $y$ de Gobierno del ALBA-TCP. Guayaquil, 30 de julio de 2013. <https://www.urjc.es/images/ceib/revista_electronica/vol_7_2013_2/ REIB_07_02_Doc04.pdf>

5 Álvaro García Linera, Geopolitica de la Amazonia. Poder hacendalpatrimonial y acumulación capitalista, La Paz, s/f. <http://www.alames.org/documentos/amazoniaAGL.pdf>

6 Fernando Coronil Ímber, El Estado Mágico. Naturaleza, Dinero y Modernidad en Venezuela, Editorial Alfa, Caracas, 2013, p. 82. 
tiende a moldear regímenes políticos caracterizados por el rentismo y el clientelismo. Genera una dependencia creciente en los sectores populares de las transferencias del Estado y tiende a debilitar sus capacidades autónomas, y con ello, la democracia. El ingreso proveniente de las actividades extractivas permite aumentar el gasto fiscal sin alterar los regímenes impositivos regresivos que caracterizan a todo el continente. La redistribución vía políticas sociales de inclusión, subsidios estatales y las transferencias monetarias directas responden a legítimas demandas de la población, pero contribuyen poco a alterar las estructuras productivas de la sociedad y las profundas desigualdades que la caracterizan. Como lo demuestra en forma contundente la experiencia de un siglo de petróleo en Venezuela, una vez instalado el extractivismo/rentismo como patrón de organización de la sociedad, éste muy difícilmente puede ser revertido.

La especialización en la producción de materias primas, lejos de permitir una acumulación que garantice la inversión en alternativas al extractivismo, tiende a bloquear la posibilidad de otras actividades generando procesos de desindustrialización en el continente, ${ }^{7}$ y el deterioro de la soberanía alimentaria. Este modelo primario-exportador representa la continuidad de las formas históricas coloniales de inserción en el mercado global basadas en la exportación de naturaleza y, como ha argumentado Joan Martínez-Alier, del comercio ecológicamente desigual. ${ }^{8}$ Con esto no se están construyendo alternativas al capitalismo, ni siquiera poniéndole obstáculos, sino alimentando su insaciable maquinaria depredadora.

El extractivismo está inseparablemente imbricado con concepciones y prácticas políticas Estado-céntricas, que como fue el caso del socialismo del siglo XX, ven al Estado como el principal agente de la transformación social. Quien formula esto con mayor claridad es Álvaro García Linera:

El Estado es el único que puede unir a la sociedad, lo único que recoge la síntesis de la voluntad general; que planea el marco estratégico y es el motor de la locomotora. El segundo es la inversión privada boliviana; tercero es la inversión extranjera; cuarto es

$7 \quad$ Pierre Salama, "China-Brasil: industrialización y 'desindustrialización temprana', Cuadernos de Economía, 31(56), 2012

8 Pablo Samaniego, María Cristina Vallejo y Joan Martínez-Alier, "Déficit comercial y déficit físico en Sudamérica", <http://www. flacsoandes.edu.ec/web/imagesFTP/1400886233.Samaniego_et_al_ Deficit_fisico_y_comercial_2014_may_20.pdf> el pequeño negocio; quinto es la economía rural; y el sexto es [la] economía indígena. Este es el orden estratégico en el que la economía del país tiene que ser estructurado. ${ }^{9}$

Esto se traduce, como en los demás países, en un proceso ya sea de planificación centralizada, o de improvisación, decidido desde arriba que deja a un lado la diversidad, la pluralidad, la posibilidad de la experimentación social, y con ello, la democracia.

El mandato político, social y económico del Plan de Desarrollo Económico y Social (PDES), es obligatorio y constituye el rector de los procesos de planificación, inversión y presupuesto para el corto y mediano plazo en los ámbitos sectorial, regional, departamental, municipal, indígena originario campesino e Institucional. ${ }^{10}$

Esta lógica Estado-céntrica tiene consecuencias. En la medida en que se han fortalecido los Estados, se han debilitado los movimientos sociales y las organizaciones autónomas. Procesos tecnocráticos-modernizantes dirigidos desde arriba, desde Estados centralizados e hiper-presidencialistas, frenan o impiden los procesos plurales de experi mentación social sin los cuales no son posibles las dinámicas de construcción colectiva de sociedades democráticas.

\section{La teoría de las ventajas comparativas y los precios de los commodities}

Retomando la teoría clásica de las ventajas comparativas en el comercio internacional, gobiernos y muchos académicos asumieron que el alza sostenida en la demanda y precio de los commodities era una indicación de que el deterioro de los términos de intercambio entre commodities y bienes industriales era cosa del pasado y que era posible, en estas nuevas condiciones, aprovechar los elevados precios de los commodities para financiar los procesos de cambio planteados. Sin embargo, en la segunda década del siglo volvieron a hacerse presentes las históricas fluctuaciones y tendencias a la

9 Álvaro García Linera, "Fue un error no liderar el pedido autonómico," Entrevista en: El Deber, Santa Cruz de la Sierra, 21 January 2007, citado por Eric Toussaint en ¿Un capitalismo andino-amazónico?, CADTM/Rebelión, 2009.

10 Ministerio de Planificación del Desarrollo. Estado Plurinacional de Bolivia. Directrices de planificación de mediano y largo plazo hacia la Agenda Patriótica 2025, Gestión 2014, pp. 5-6 
baja de los precios de los commodities en el mercado internacional, afectando cada uno de los principales renglones exportados por el continente.

En el segundo semestre del 2014, el precio del petróleo bajó más de 50\%; entre mediados del 2011 y finales del 2014; el precio del cobre se redujo en 35\%; el precio del mineral de hierro en noviembre 2014 era menos del 40\% del precio de febrero del 2011; entre junio y octubre del 2014 el precio de la soya bajó en un $27 \% .{ }^{11} \mathrm{El}$ país más afectado ha sido Venezuela ya que el petróleo representó en los últimos años el 96\% del valor de sus exportaciones. Para el conjunto de América del Sur, de acuerdo al Banco Interamericano de Desarrollo, el valor total de las exportaciones tuvo una baja de $17 \%$ en el primer semestre del año 2015, comparado con el mismo período del ańo anterior. ${ }^{12}$

Termina así el corto ciclo (menos de una década), de los elevados precios de los commodities. La continuidad de políticas sociales y redistributivas de estos años está lejos de estar garantizada. ¿Estamos igualmente en presencia del fin del ciclo de los gobiernos progresistas en el continente que se hicieron tan dependientes de este ciclo de los commodities?

El respaldo de la población a los gobiernos denominados progresistas no es hoy, obviamente, lo que era hace muy pocos años. Estos gobiernos tuvieron más éxito en la creación de nuevos consumidores que en el fortalecimiento del tejido asociativo/participativo de estas sociedades y su politización, o en la preparación de los sectores populares para defender sus conquistas ante la inevitable reacción de la derecha y de los intereses imperiales en contra de todo intento de transformación de estas sociedades. En diferentes grados, cada uno de estos gobiernos se ha caracterizado por severos déficits democráticos.

Los gobiernos de Ecuador y Bolivia han sufrido una significativa baja en el apoyo de la población en las últimas elecciones regionales y municipales. Dilma Rousseff ganó la re-elección en el año 2015 por muy estrecho margen. En una sociedad extraordinariamente dividida, una derecha corrupta con control total del parlamento condujo un proceso de impeachment que culminó con su destitución y su reemplazo por Michel Temer, quien en forma acelerada ha tomado pasos en la dirección de revertir

11 Nasdaq <www.nasdaq.com/markets/>

12 Javier Lewkowicz, "Fuerte caída de las exportaciones de los países de América del Sur. Viento de frente para la región", Página 12, Buenos Aires, 17 de octubre 2015. aspectos centrales de las políticas del PT. Entre otras medidas se ha anunciado la reducción del papel de Petrobras en el control de las reservas petroleras del país. Los resultados de las elecciones municipales realizadas poco tiempo después confirmaron un severo retroceso no solo del PT, sino del conjunto de la izquierda brasileña.

El candidato del kirchnerismo fue derrotado en la segunda vuelta de las elecciones presidenciales del año 2015 por el empresario neoliberal Mauricio Macri. Por primera vez en la historia de Argentina, la derecha llega al gobierno por elecciones, no por la vía de costumbre, los golpes militares. Como en el caso de Brasil, ha comenzado un acelerado proceso de reversión de las políticas sociales populares del gobierno anterior.

El gobierno venezolano, que perdió las elecciones parlamentarias de fines del 2015 por una amplia mayoría, ha hecho todo lo posible por impedir la realización del referéndum revocatorio que está contemplado en la constitución, al tanto de que no tiene posibilidad alguna de ganar esta consulta.

Los procesos de integración regional, que poco avanzaron en el ámbito productivo durante estos años, están hoy en franco retroceso. La denominada "integración abierta”, el acercamiento a la Alianza del Pacífico y la disposición a retomar las negociaciones para la firma de un acuerdo de libre comercio entre Mercosur y la Unión Europea definen las orientaciones principales de los nuevos gobiernos de Argentina y Brasil.

\section{Formas de inserción en el mercado mundial y relaciones con China}

La superación del capitalismo y el camino hacia sociedades del buen vivir, capaces de vivir en armonía con la naturaleza, exige, necesariamente, procesos tendenciales de autonomización y desacoplamiento respecto a los mecanismos de mercantilización que caracterizan al capital, y la creación de otros tejidos sociales y espacios productivos sustentados en alternativas al crecimiento sin fin, así como otros imaginarios y otros patrones culturales de consumo. Ello solo sería posible al interior de espacios de integración de creciente densidad sustentados en esas otras lógicas sociales. Esto no es compatible con modelos productivos basados en el extractivismo y economías primario-exportadoras cuya prioridad es el acceso a mercados extra 
continentales. ${ }^{13}$ Con el vivir bien/buen vivir no se busca un capitalismo más humano, ni un desarrollo más sostenible. Implica, por el contrario, una crítica radical a la linealidad del desarrollo histórico y a la idea misma de desarrollo.

Las relaciones con China, lejos de reducir la dependencia del continente respecto al mercado capitalista global y sus patrones culturales, la ha profundizado. La extraordinaria demanda china de materias primas produjo alzas muy importantes tanto en la demanda como en los precios de los principales commodities que produce el continente, ${ }^{14}$ empujando hacia la reprimarización de sus economías. Mientras que, para el conjunto de América Latina, las materias primas representan un poco más de $40 \%$ del total de las exportaciones, la cifra corres pondiente a sus exportaciones a China es de prácticamente $70 \%$. En lo fundamental, en sus relaciones comerciales con China, América Latina intercambia commodities por bienes industriales. ${ }^{15}$

Una elevada proporción del valor total de las exportaciones de los países suramericanos a China está concentrada en solo un, dos o tres productos básicos de origen primario extractivo o alguna manufactura de origen agropecuario: petróleo, mineral de hierro, cobre, soya, harina de soya. En el caso de Brasil, el país más industrializado del continente, entre los años 1995 y el 2008, el peso relativo de los productos primarios en las exportaciones totales a China pasó de $20 \%$ a $80 \%$, principalmente mineral de hierro y soya. ${ }^{16}$ Esta especialización primario exportadora está inducida igualmente por los créditos y las inversiones chinas. Desde el año 2005 China ha otorgado más de 100 mil millones de dólares en créditos al continente, un volumen mucho mayor de los créditos combinados provenientes del Banco Mundial, del Banco Interamericano de Desarrollo y el Banco de Exportación e Importación de los Estados Unidos. ${ }^{17}$ La mayor parte de estos créditos están directamente asociados a la producción de com-

13 Ariel Slipak, La expansión de China en América Latina: incidencia en los vínculos comerciales argentino-brasileros, Observatorio de la Política China,

$<$ http://www.politica-china.org/nova.php?id=5248\&clase $=26 \& l g=$ gal $>$

14 Rhys Jenkins, "El 'efecto China' en los precios de los productos básicos y en el valor de las exportaciones de América Latina”, Revista CEPAL, $\mathrm{n}^{\circ} 103$, abril 2011.

15 CEPAL, Promoción del comercio y la inversión con China Desafíos y oportunidades en la experiencia de las cámaras empresariales latinoamericanas, Santiago de Chile, 2013

16 Mónica Bruckmann, Recursos naturales y la geopolítica de la integración sudamericana, <http://alainet.org/active/45772>

17 Interamerican Dialogue. China-Latin America Finance Database, $<$ http://www.thedialogue.org/map_list> modities, o a las infraestructuras requeridas para ello. En algunos casos, como Venezuela y Ecuador, parte de estos créditos deben ser pagados directamente con petróleo. ${ }^{18}$ Las inversiones chinas están igualmente concentradas en actividades extractivas. ${ }^{19}$ Todo esto obliga a seguir por la senda del extractivismo depredador a largo plazo.

A comienzos del año 2016, el gobierno venezolano, ante el colapso de los precios del petróleo y en condiciones de una muy profunda crisis fiscal, ante las perspectiva del agotamiento del modelo petrolero rentista que ha definido al país durante ya un siglo, ha declarado la apertura de $12 \%$ del territorio nacional a las empresas transnacionales para la explotación minera en gran escala. Con este macro proyecto, denominado el Arco Minero del Orinoco, se busca reemplazar el rentismo petrolero por el rentismo minero. Se trata de un modelo tan depredador como la explotación de hidrocarburos que no solo implicaría efectos devastadores sobre los bosques, la biodiversidad, las aguas, la producción hidroeléctrica de esta vasta zona, sino que amenaza con el etnocidio de los pueblos indígenas habitantes de dicho territorio. Estas decisiones las toma un gobierno por decreto un gobierno de tendencias cada vez más autoritario, al margen de la Constitución y de toda consulta a la población venezolana.

\section{Más allá del extractivismo: la transición}

Ante el avance incontenible de estas lógicas depredadoras, en estos años no solo han continuado, sino que se han profundizado y extendido en todo el continente las luchas y resistencias en contra del extractivismo y de sus infraestructuras (represas, carreteras oleoductos, puertos). Se han articulado redes continentales contra la mega minería, la explotación petrolera, las grandes represas hidroeléctricas, los monocultivos y los transgénicos. Pueblos indígenas, afrodescendientes y campesinos, así como habitantes de pequeńas ciudades alejadas de las metrópolis, son hoy los principales protagonistas de estas luchas. Se han logrado importantes victorias

18 Kevin P. Gallagher, Amos Irwin y Katherine Koleski “¿Un mejor trato? Análisis comparativo de los préstamos chinos en América Latina", Universidad Nacional Autónoma de México, Facultad de Economía, Centro de Estudios China-México. Número 1, 2013.

19 Enrique Dussel Peters. Chinese FDI in Latin America: Does Ownership Matter? Working Group on Development and Environment in the Americas.

$<$ http://ase.tufts.edu/gdae/Pubs/rp/DP33DusselNov12.pdf> 
locales y en muchas ocasiones las empresas se han tenido que retirar ante la resistencia de las poblaciones afectadas.

Sin embargo, en el contexto de una cultura que es no solo dominante, sino que ha sido reforzada por las políticas de los gobiernos progresistas, estas luchas están hoy en condiciones de profunda desventaja. Mientras la confianza en el desarrollo siga siendo hegemónica tanto en la derecha como en la izquierda y mientras los impactos depredadores del extractivismo sigan estando lejos de los centros metropolitanos que concentran a la mayoría de la población, será difícil que estas resistencias sean asumidas más ampliamente, sobre todo por los sectores populares urbanos que han sido beneficiados por las políticas sociales financiadas por el extractivismo.

Hoy en el continente nadie está proponiendo que de un día para otro se decrete el fin del extractivismo y que a partir de esa fecha no se extraiga un barril de petróleo, ni una tonelada de mineral de hierro, ni se siembre una hectárea de soya transgénica. Sí se exige, eso sí, que se detenga en forma inmediata la expansión de estas actividades. Es urgente ampliar y profundizar los debates y los procesos de experimentación sobre la necesaria transición hacia una economía no-extractivista, no-rentista, más allá de la retórica vacía sobre su necesidad que suele estar presente en los discursos gubernamentales. ¿Qué medidas concretas habría que tomar en el presente en asuntos tan medulares como el energético, la producción de alimentos, o el modelo de transporte, la urbanización, para dar pasos en la dirección de otro patrón productivo, (y un modelo de sociedad) no extractivista, no-rentista? ${ }^{20}$ ¿Cómo crear condiciones favorables para dinámicas de experimentación social sin las cuales no hay transformación democrática posible? $\mathrm{Al}$ no haber iniciado esta transición, los llamados gobiernos progresistas pasarán a la historia como los responsables de haber acelerado los procesos de destrucción del planeta y de haber contribuido a frustrar las esperanzas de otro mundo posible.

20 Ver, por ejemplo: Alberto Acosta, Esperanza Martínez, William Sacher, "Salir del extractivismo: una condición para el Sumak Kawsay. Propuestas sobre petróleo, minería y energía en el Ecuador", en Miriam, Lang y otras, Alternativas al capitalismo/colonialismo del siglo XXI, Fundación Rosa Luxemburg, Quito, 2013; Eduardo Gudynas, "Debates sobre el desarrollo y sus alternativas en América Latina: Una breve guía heterodoxa", en Miriam Lang y Dunia Mokrani (compiladoras), Más allá del desarrollo, Fundación Rosa Luxemburg, Quito, 2011. 